\title{
Miniaturized Tables of Bessel Functions. II $^{*}$
}

\author{
By Yudell L. Luke
}

\begin{abstract}
In a previous study, we discussed the expansion of two-parameter functions in a double series of Chebyshev polynomials, and, in particular, we presented coefficients for the evaluation of the modified Bessel function $(2 z / \pi)^{1 / 2} e^{z} K_{\nu}(z)$ to 20 decimals for all $z \geqq 5$ and all $\nu, 0 \leqq \nu \leqq 1$. In the present study, we give similar coefficients for the evaluation of $g e^{-z} z^{-\mu} I_{\nu}(z)$ to at least 20 decimals where $I_{\nu}(z)$ is the modified Bessel function of the first kind and $g$ and $\mu$ are certain constants which depend on the range of the parameter and variable for four different situations. The ranges are (1) $0<z \leqq 8,0 \leqq \nu \leqq 4$; (2) $0<z \leqq 8,4 \leqq \nu \leqq 8 ;(3) z \geqq 8,-1 \leqq \nu \leqq 0 ;(4) z \geqq 8,0 \leqq \nu \leqq 1$.
\end{abstract}

1. Introduction. In a previous study [1], we discussed the expansion of twoparameter functions in a double series of Chebyshev polynomials, and, in particular, we presented coefficients for the evaluation of the modified Bessel function $(2 z / \pi)^{1 / 2}$ $\times e^{z} K_{\nu}(z)$ to 20 decimals for all $z \geqq 5$ and all $\nu, 0 \leqq \nu \leqq 1$. Since $K_{\nu}(z)=K_{-\nu}(z)$ and $K_{v}(z)$ satisfies a three-term recurrence formula which is stable in the forward direction, we have in essence coefficients for the evaluation of $K_{\nu}(z)$ for all $z \geqq 5$ and all $\nu \geqq 0$.

In the present study, we give similar coefficients for the evaluation of $g e^{-z} z^{-\mu} I_{\nu}(z)$ to at least 20 decimals where $I_{\nu}(z)$ is the modified Bessel function of the first kind and $g$ and $\mu$ are certain constants which depend on the range of the parameter and variable for four different situations as follows.

$$
\begin{aligned}
& z \text { range } \\
& \nu \text { range } \\
& \mu \quad g \\
& 0<z \leqq 8 \\
& 0 \leqq \nu \leqq 4 \\
& \nu \\
& 1 \\
& 0<z \leqq 8 \\
& 4 \leqq \nu \leqq 8 \\
& \nu \\
& 1 \\
& z \geqq 8 \\
& -1 \leqq \nu \leqq 0 \\
& -\frac{1}{2} \\
& (2 \pi)^{-1 / 2} \\
& z \geqq 8 \\
& 0 \leqq \nu \leqq 1 \\
& -\frac{1}{2} \\
& (2 \pi)^{-1 / 2}
\end{aligned}
$$

The recursion formula for $I_{\nu}(z)$ is always stable in the backward direction but only conditionally stable in the forward direction. Thus, even with the coefficients given here, we still lack coefficients to compute $e^{-z} I_{\nu}(z)$ for all real $z$ and for $\nu$ sufficiently large. A study to correct this deficiency is under way and will be reported at a later date.

2. Chebyshev Expansions for $I_{\nu}(z)$. In [2, Vol. 2, pp. 338-340, 359-367], we gave coefficients for the expansion of $z^{-1} I_{\nu}(z)$ in series of Chebyshev polynomials for

Received April 27, 1971.

AMS 1969 subject classifications. Primary 3325, 4216, 6505, 6525.

Key words and phrases. Bessel functions, approximation of bivariate functions, expansions in double series of Chebyshev polynomials, mathematical tables.

* This research was supported by the United States Atomic Energy Commission under Contract AT(11-1)1619. 
$0<z \leqq 8, \nu=0, \pm \frac{1}{4}, \pm \frac{1}{3}, \pm \frac{1}{2}, \pm \frac{2}{3}, \pm \frac{3}{4}, 1$, and, similarly, for the expansion of $(2 \pi z)^{-1 / 2} e^{-z} I_{\nu}(z)$ for $z \geqq 8, \nu=0, \frac{1}{4}, \frac{1}{3}, \frac{2}{3}, \frac{3}{4}, 1$. The coefficients for the range $0<z \leqq 8$ are based on the ${ }_{0} F_{1}$ representation for $I_{v}(z)$ which does not directly reflect the fact that for fixed $\nu, I_{\nu}(z)$ grows exponentially with $z$ as $z$ increases in the sector $|\arg z|<$ $\pi / 2$. Now, $I_{v}(z)$ has a representation in terms of $a_{1} F_{1}$ which does reflect this exponential behavior and, in this present paper, development of the desired coefficients is based on this representation for $0<z \leqq 8$. The representation used in the cited reference for $z \geqq 8$ is also used in our present study to derive the desired coefficients already noted.

From [2, Vol. 1, p. 213], we have

$$
\begin{aligned}
z^{-\nu} e^{-z} I_{\nu}(z) & =\left[2^{\nu} \Gamma(\nu+1)\right]^{-1}{ }_{1} F_{1}(a ; c ;-2 z), \\
a & =c / 2=\frac{1}{2}+\nu .
\end{aligned}
$$

In general, from [2, Vol. 2, p. 35],

$$
\begin{aligned}
&{ }_{1} F_{1}(a ; c ; z)=\sum_{k=0}^{\infty} G_{k}(a, c, \lambda) T_{k}^{*}(z / \lambda), \\
& G_{k}(a, c, \lambda)=\frac{\epsilon_{k}(a)_{k} \lambda^{k}}{2^{2 k}(c)_{k} k !}{ }_{2} F_{2}\left(\begin{array}{ll}
a+k, & \frac{1}{2}+k \\
c+k, & 1+2 k
\end{array} \mid \lambda\right), \\
& \frac{2 G_{k}(a, c, \lambda)}{\epsilon_{k}}= \frac{(k+1)}{(k+a)}\left\{-\frac{(k+3-a)}{(k+2)}+\frac{4(k+c)}{\lambda}\right\} G_{k+1}(a, c, \lambda) \\
&+\frac{2}{(k+a)}\left\{\frac{1}{2}(k+a)+\frac{2(k+1)(k+3-c)}{\lambda}\right\} G_{k+2}(a, c, \lambda) \\
&+\frac{(k+1)(k+3-a)}{(k+2)(k+a)} G_{k+3}(a, c, \lambda) .
\end{aligned}
$$

In the above,

$$
\epsilon_{k}=1 \quad \text { if } k=0, \quad \epsilon_{k}=2 \quad \text { if } k>0 .
$$

Using [2, Vol. 1, p. 244], we find that for $a, c$ and $\lambda$ fixed,

$$
\begin{aligned}
G_{k}(a, c, \lambda)= & \frac{\Gamma(c)(\lambda / 4)^{k} k^{a-c}}{\Gamma(a) k !} \\
& \cdot\left[1+\frac{\lambda^{2}-8 \lambda(c-a)-8(c-a)(c+a-1)}{16 k}+O\left(k^{-2}\right)\right] .
\end{aligned}
$$

Thus, the expansion formula (2) converges and since the ${ }_{1} F_{1}$ in (2) is one when $z=0$, it follows that

$$
\sum_{k=0}^{\infty}(-)^{k} G_{k}(a, c, \lambda)=1 .
$$

Further, after the manner of the discussion given in [2, Vol. 2, pp. 159-166], we can show that use of the recursion formula (4) in the backward direction is convergent. Thus, for a fixed $\lambda$, we can generate the coefficients $G_{k}(a, c, \lambda)$ for given values of $a$ and $c$. Suppose for example that $c$ is fixed and we permit $a$ to vary. Then, we can find coefficients $D_{r, k}(c, \lambda)$ such that 


$$
G_{k}(a, c, \lambda)=\sum_{r=0}^{\infty} D_{r, k}(c, \lambda) T_{r}^{*}(a / \omega), \quad 0 \leqq a \leqq \omega,
$$

and so achieve a double series of Chebyshev polynomials for the evaluation of ${ }_{1} F_{1}(a ; c ; z)$ for $c$ fixed, valid for $0 \leqq z \leqq \lambda$ and $0 \leqq a \leqq \omega$. The manner of getting $D_{r, k}(c, \lambda)$ has been given in [1] and we omit further details.

Next, we seek a descending-type expansion in series of Chebyshev polynomials for the evaluation of $I,(z)$ in the neighborhood of $z=+\infty$. To this end, we can write [2, Vol. 1, p. 226, Eq. (9)], [2, Vol. 2, p. 22, Eq. (10)],

$$
\begin{aligned}
& I_{\nu}(z)=(2 \pi z)^{-1 / 2} e^{z} F_{\nu}(z), \\
& F_{\nu}(z)=G_{1,2}^{1,1}\left(\begin{array}{l|l}
2 z & \begin{array}{l}
1 \\
\frac{1}{2}+\nu,
\end{array} \\
\frac{1}{2}-\nu
\end{array}\right), \\
& F_{\nu}(z)=\sum_{k=0}^{\infty} M_{k}(\nu, \lambda) T_{k}^{*}(\lambda / z), \quad \lambda \text { fixed, } \lambda / z \leqq 1, z>0, \\
& M_{k}(\nu, \lambda)=\pi^{-1 / 2} \epsilon_{k}(-)^{k} G_{2,3}^{2,1}\left(\begin{array}{l|l}
2 \lambda & \begin{array}{l}
1-k, k+1 \\
\frac{1}{2}, \quad \frac{1}{2}+\nu,
\end{array} \frac{1}{2}-\nu
\end{array}\right), \\
& M_{k}(\nu, \lambda)=\pi^{-1 / 2} \epsilon_{k}(-)^{k} G_{3,2}^{1,2}\left(\frac{1}{2 \lambda} \mid \begin{array}{lll}
\frac{1}{2}, & \frac{1}{2}-\nu, & \frac{1}{2}+\nu \\
k, & -k
\end{array}\right),
\end{aligned}
$$

and from [2, Vol. 2, pp. 153, 154 and Remark 1, p. 155], we have the recursion formula

$$
\begin{aligned}
\frac{2 M_{k}(\nu, \lambda)}{\epsilon_{k}}= & 2(k+1)\left\{1-\frac{(2 k+3)(k+3 / 2+\nu)(k+3 / 2-\nu)}{2(k+2)\left(k+\frac{1}{2}+\nu\right)\left(k+\frac{1}{2}-\nu\right)}\right. \\
& \left.+\frac{8 \lambda}{\left(k+\frac{1}{2}+\nu\right)\left(k+\frac{1}{2}-\nu\right)}\right\} M_{k+1}(\nu, \lambda) \\
& +\left\{1-\frac{2(k+1)(2 k+3+4 \lambda)}{\left(k+\frac{1}{2}+\nu\right)\left(k+\frac{1}{2}-\nu\right)}\right\} M_{k+2}(\nu, \lambda) \\
& -\frac{(k+1)(k+5 / 2+\nu)(k+5 / 2-\nu)}{(k+2)\left(k+\frac{1}{2}+\nu\right)\left(k+\frac{1}{2}-\nu\right)} M_{k+3}(\nu, \lambda), \quad k \geqq 0 .
\end{aligned}
$$

Actually, (13) is not valid if $\nu$ is half an odd integer unless $k+\frac{1}{2}-\nu>0$. If, for example, $\nu=\frac{1}{2}+n,(13)$ is only valid for $k>n$. However, we can get a further relation if first we multiply through by $k+\frac{1}{2}-\nu$ and then set $k+\frac{1}{2}-\nu=0$ for $k=n$. In particular, if $\nu=\frac{1}{2}$, we have

$$
\begin{aligned}
& \frac{2 M_{k}\left(\frac{1}{2}, \lambda\right)}{\epsilon_{k}}= {\left[\frac{8 \lambda-3(k+1)}{k}\right] M_{k+1}\left(\frac{1}{2}, \lambda\right)-\left[\frac{8 \lambda+3(k+2)}{k}\right] M_{k+2}\left(\frac{1}{2}, \lambda\right) } \\
&-\frac{(k+3)}{k} M_{k+3}\left(\frac{1}{2}, \lambda\right), \quad k>0 \\
&(8 \lambda-3) M_{1}\left(\frac{1}{2}, \lambda\right)=(8 \lambda+6) M_{2}\left(\frac{1}{2}, \lambda\right)+3 M_{3}\left(\frac{1}{2}, \lambda\right) .
\end{aligned}
$$

It can be shown that

$$
M_{0}\left(\frac{1}{2}, \lambda\right)=2 \pi^{-1 / 2} \operatorname{Erf}(x), \quad x^{2}=2 \lambda, \quad \operatorname{Erf}(x)=\int_{0}^{x} e^{-t^{2}} d t
$$




$$
M_{1}\left(\frac{1}{2}, \lambda\right)=8 \lambda\left[1-M_{0}\left(\frac{1}{2}, \lambda\right)\right]-4(2 \lambda / \pi)^{1 / 2} e^{-2 \lambda} .
$$

With $z \rightarrow+\infty$, (11) yields the useful normalization equation

$$
\sum_{k=0}^{\infty}(-)^{k} M_{k}(\nu, \lambda)=1
$$

From [2, Vol. 2, pp. 23, 24],

$$
M_{k}(\nu, \lambda) \sim k^{-1}\left[u \exp \left\{-3\left(2 \lambda k^{2} e^{i \pi}\right)^{1 / 3}\right\}+v \exp \left\{-3\left(2 \lambda k^{2} e^{-i \pi}\right)^{1 / 3}\right\}\right]
$$

where $u$ and $v$ are constants. The two other linearly independent solutions of (14) can be taken in a form such that they are

$$
O\left(k^{-1} \exp \left\{3\left(2 \lambda k^{2}\right)^{1 / 3}\right\}\right) \text { and } O\left(k^{-1} \exp \left\{-3\left(2 \lambda k^{2} e^{i \pi}\right)^{1 / 3}\right\}\right) .
$$

It follows that the desired solution of (14) is not minimal in the sense of Gautschi [3] or not antidominant in the sense of Wimp [4], and consequently the backward recursion process for the evaluation of $M_{k}(\nu, \lambda)$ will fail unless modified. The necessary modification is discussed in [2, Vol. 2, pp. 163-164] and studied further in Wimp [4, Theorem 3]. We now describe this procedure.

Let $N$ be a large positive integer. Put

$$
g_{N+k}^{(N)}=0, \quad k=2,3, \cdots, \quad g_{N+1}^{(N)}=1
$$

and compute $g_{n}^{(N)}, n=N, N-1, \cdots, 0$ from (13) with $M_{k}(\nu, \lambda)$ replaced by $g_{k}^{(N)}$. (Here we assume that $\nu$ is not half an odd integer. The case when $\nu=\frac{1}{2}$ is treated later.) Put

(22) $M_{n}^{(N)}(\nu, \lambda)=\rho^{(N)} g_{n}^{(N)}, \quad n=0,1, \cdots, N+1, \quad \rho^{(N)}=\left(\sum_{n=0}^{N+1}(-)^{n} g_{n}^{(N)}\right)^{-1}$.

Let $N_{1}, N_{2}$ be two different $N$ values. We can find a number $\mu$ depending on $N_{1}$ and $N_{2}$ such that

$$
\mu \sum_{k=0}^{N_{1}+1} M_{k}^{\left(N_{1}\right)}(\nu, \lambda)+(1-\mu) \sum_{k=0}^{N_{2}+1} M_{k}^{\left(N_{2}\right)}(\nu, \lambda)=(2 \pi \lambda)^{1 / 2} e^{-\lambda} I_{\nu}(\lambda) .
$$

Then

$$
\begin{aligned}
\lim _{N_{1} \rightarrow \infty ; N_{2} \rightarrow \infty ; N_{1} \neq N,}\left[\mu M_{k}^{\left(N_{1}\right)}(\nu, \lambda)+(1-\mu) M_{k}^{\left(N_{2}\right)}(\nu, \lambda)\right]=M_{k}(\nu, \lambda), & \\
k & =0,1, \cdots .
\end{aligned}
$$

If $\nu$ is half an odd integer, another technique must be used as the process just described breaks down due to the presence of the product $\left(k+\frac{1}{2}+\nu\right)\left(k+\frac{1}{2}-\nu\right)$. To illustrate, consider the case $\nu=\frac{1}{2}$. In this event,

$$
F_{1 / 2}(z)=1-e^{-2 z} \text {. }
$$

We have need for the three normalization relations

$$
\begin{aligned}
1-e^{-2 \lambda} & =\sum_{k=0}^{\infty} M_{k}\left(\frac{1}{2}, \lambda\right) \\
1 & =\sum_{k=0}^{\infty}(-)^{k} M_{k}\left(\frac{1}{2}, \lambda\right),
\end{aligned}
$$




$$
1-e^{-4 \lambda}=\sum_{k=0}^{\infty}(-)^{k} M_{2 k}\left(\frac{1}{2}, \lambda\right),
$$

which come from (9)-(11) when $\nu=\frac{1}{2}$ and $z=2 \lambda,+\infty$ and $4 \lambda$, respectively.

Again, let $N$ be a large positive integer, set

$$
g_{N+k}^{(N)}=0, \quad k=2,3, \cdots, \quad g_{N+1}^{(N)}=1,
$$

and compute

$$
g_{n}^{(N)}, \quad n=N, N-1, \cdots, 1,
$$

from (13) with $M_{k}\left(\frac{1}{2}, \nu\right)$ replaced by $g_{k}^{(N)}$. Let

$$
M_{k}^{(N)}=\rho^{(N)} g_{k}^{(N)}, \quad k=1,2, \cdots, \quad M_{0}^{(N)}=g_{0}^{(N)} .
$$

Then from (26) and (27), respectively, we have

$$
\begin{aligned}
g_{0}^{(N)}+\rho^{(N)} \sum_{k=1}^{\infty} g_{k}^{(N)} & =1-e^{-2 \lambda}, \\
g_{0}^{(N)}+\rho^{(N)} \sum_{k=1}^{\infty}(-)^{k} g_{k}^{(N)} & =1 .
\end{aligned}
$$

Thus

$$
\rho^{(N)}=\frac{-e^{-2 \lambda}}{2 \sum_{k=0}^{\infty} g_{2 k+1}^{(N)}}
$$

and $g_{0}^{(N)}$ can be recovered from either (31) or (32). Let $N_{1}, N_{2}$ be two different $N$ numbers. We can find a number $\mu$ depending on $N_{1}$ and $N_{2}$ such that

$$
\mu \sum_{k=0}^{N_{1}+1}(-)^{k} M_{2 k}^{\left(N_{1}\right)}\left(\frac{1}{2}, \lambda\right)+(1-\mu) \sum_{k=0}^{N_{2}+1}(-)^{k} M_{2 k}^{\left(N_{2}\right)}\left(\frac{1}{2} \lambda\right)=1-e^{-4 \lambda} .
$$

Then

$$
\begin{aligned}
\lim _{N_{1} \rightarrow \infty ; N_{2} \rightarrow \infty ; N_{1} \neq N_{2}}\left[\mu M_{k}^{\left(N_{1}\right)}\left(\frac{1}{2}, \nu\right)+(1-\mu) M_{k}^{\left(N_{3}\right)}\left(\frac{1}{2}, \nu\right)\right]=M_{k}\left(\frac{1}{2}, \lambda\right), \\
k=0,1, \ldots .
\end{aligned}
$$

The coefficients can be checked using (16) and (17). Alternatively, we can make use of (17) to find a number $\mu^{*}$ such that

$$
\begin{aligned}
& 8 \lambda\left[\mu^{*} M_{0}^{\left(N_{1}\right)}\left(\frac{1}{2}, \lambda\right)+\left(1-\mu^{*}\right) M_{0}^{\left(N_{2}\right)}\left(\frac{1}{2}, \lambda\right)\right] \\
& +\mu^{*} M_{1}^{\left(N_{1}\right)}\left(\frac{1}{2}, \lambda\right)+\left(1-\mu^{*}\right) M_{1}^{\left(N_{3}\right)}\left(\frac{1}{2}, \lambda\right)=8 \lambda-4(2 \lambda / \pi)^{1 / 2} e^{-2 \lambda} .
\end{aligned}
$$

Then $M_{k}\left(\frac{1}{2}, \lambda\right)$ follows as in (35) with $\mu$ replaced by $\mu^{*}$ and (28) can be used as a check.

Another scheme to compute $M_{k}(\nu, \lambda)$ for $\nu$ half an odd integer is to use the procedure described by (21)-(24) to get $M_{k}(\nu, \lambda)$ for $\nu$ in the neighborhood of half an odd integer and then employ the Lagrangian interpolation formula.

3. Numerical Results. From (1), (2) and (8), with a slight change of notation, we have 


$$
\begin{aligned}
I_{\nu}(z)=z^{\nu} e^{z} \sum_{k=0}^{\infty} H_{k}(\nu) T_{k}^{*}(z / 8), & 0<z \leqq 8, \\
H_{k}(\nu)=\sum_{r=0}^{\infty} D_{r, k} T_{r}^{*}\left(\frac{\nu-s}{t}\right), & s \leqq \nu \leqq s+t .
\end{aligned}
$$

In Tables 1 and 2 of the microfiche section we present values of $D_{r, k}$ which were evaluated by the technique described in [1] for $s=0, t=4$ and $s=t=4$, respectively. To develop the numerics, values of $\Gamma(\nu+1)$ were required. These were obtained by use of the schema of my previous paper [5]. Numerous checks were made on the coefficients. In addition to those of the kind discussed in [1], checks were also made using the recurrence formula for $I_{\nu}(z)$, namely

$$
I_{p+1}(z)+\frac{2 \nu}{z} I_{\nu}(z)-I_{\nu-1}(z)=0 .
$$

Further checks were accomplished by comparing values deduced from (37) with those computed from power series, especially when $\nu$ is half an odd integer, for in this instance

$$
\begin{aligned}
e^{-z} I_{n+1 / 2}(z) & =(2 \pi z)^{-1 / 2}\left[A_{n}(z)+(-)^{n+1} e^{-2 z} A_{n}(-z)\right], \\
A_{n}(z) & ={ }_{2} F_{0}\left(-n, n+1 ; \frac{1}{2 z}\right),
\end{aligned}
$$

and $A_{n}(z)$ is a polynomial in $z^{-1}$ of degree $n$. Wronskian relations were also used to get checks. The computations were designed so that the coefficients for $0 \leqq \nu \leqq 4$ are accurate to about 25D while those for $4 \leqq \nu \leqq 8$ are accurate to about 27D. To evaluate $e^{-z} I_{\nu}(z)$, we must incorporate the value of $z^{\nu}$. As $0<z \leqq 8$, we see that the coefficients are sufficiently accurate to produce $e^{-z} I_{v}(z)$ to about 20 decimals at least.

From (9)-(11) with a slight change of notation we write

$$
\begin{array}{rlrl}
I_{\nu}(z) & =(2 \pi z)^{-1 / 2} e^{z} \sum_{k=0}^{\infty} M_{k}(\nu) T_{k}^{*}(8 / z), & & z \geqq 8, \\
M_{k}(\nu) & =\sum_{r=0}^{\infty} E_{r, k} T_{r}^{*}(\nu), & 0 \leqq \nu \leqq 1, \\
M_{k}(\nu)=\sum_{r=0}^{\infty} F_{r, k} T_{r}^{*}(-\nu), & -1 \leqq \nu \leqq 0 .
\end{array}
$$

In Tables 3 and 4 of the microfiche section we give values of $E_{r, k}$ and $F_{r, k}$, respectively. In the development of these coefficients, the appropriate values of $(2 \pi \lambda)^{1 / 2} e^{-\lambda} I_{v}(\lambda)$, as required by (23), were obtained from (37) for $\nu>0$ and (38) was used to get the values needed for $\nu<0$. Again, the coefficients were subjected to numerous checks. For example, for $z=8$, we compared values of $I_{v}(z)$, as obtained from (40)-(42), with those obtained from (37) and (38) when appropriate. We also used the defining relation for $K_{v}(z)$ in terms of $I_{\nu}(z)$ and $I_{-\nu}(z)$ to compare values obtained using the coefficients in [1] and the coefficients in the present tables. Further checks were gotten by use of a Wronskian relation. The coefficients are sufficiently accurate to enable the computation of $e^{-z}(2 \pi z)^{1 / 2} I_{v}(z)$ to about 22 decimals. 
4. Acknowledgment. I am indebted to Miss Rosemary Moran for her aid in the development of the coefficients.

Department of Mathematics

University of Missouri

Kansas City, Missouri 64110

1. Y. L. LUKE, "Miniaturized tables of Bessel functions," Math. Comp., v. 25, 1971, pp. 323-330

2. Y. L. LUKe, The Special Functions and Their Approximations, Vols. I, II, Math. in Science and Engineering, vol. 53, Academic Press, New York, 1969. MR 39 \#3039; MR 40 \#2909.

3. W. GAUTSCHI, "Computational aspects of three-term recurrence relations," SIAM Rev., v. 9, 1967, pp. 24-82. MR 35 \#3927.

4. J. WIMP, Recent Developments in Recursive Computation, Aerospace Research Laboratories, Report ARL 69-0104, Wright-Patterson Air Force Base, Ohio, 1969.

5. Y. L. LUKE, "Evaluation of the Gamma function by means of Padé approximations," SIAM J. Math. Anal., v. 1, 1970, pp. 266-281. 